\title{
IN SITU CONDITIONS AFFECTING THE DUCTILITY CAPACITY OF LIGHTLY REINFORCED CONCRETE WALL STRUCTURES IN THE CANTERBURY EARTHQUAKE SEQUENCE
}

\author{
Gareth J. Morris ${ }^{1}$, Desmond K. Bull ${ }^{2}$ and Brendon A. Bradley ${ }^{3}$
}

(Submitted December 2014; Reviewed April 2015; Accepted July 2015)

\begin{abstract}
Following the 2010-2011 Canterbury (New Zealand) earthquake sequence, lightly reinforced wall structures in the Christchurch central business district were observed to form undesirable crack patterns in the plastic hinge region, while yield penetration either side of cracks and into development zones was less than predicted using empirical expressions. To some extent this structural behaviour was unexpected and has therefore demonstrated that there may be less confidence in the seismic performance of conventionally designed reinforced concrete $(\mathrm{RC})$ structures than previously anticipated. This paper provides an observation-based comparison between the behaviour of RC structural components in laboratory testing and the unexpected structural behaviour of some case study buildings in Christchurch that formed concentrated inelastic deformations. The unexpected behaviour and poor overall seismic performance of 'real' buildings (compared to the behaviour of laboratory test specimens) was due to the localization of peak inelastic strains, which in some cases has arguably led to: (i) significantly less ductility capacity; (ii) less hysteretic energy dissipation; and (iii) the fracture of the longitudinal reinforcement. These observations have raised concerns about whether lightly reinforced wall structures can satisfy the performance objective of "Life Safety" at the Ultimate Limit State. The significance of these issues and potential consequences has prompted a review of potential problems with the testing conditions and procedures that are commonly used in seismic experimentations on RC structures. This paper attempts to revisit the principles of RC mechanics, in particular, the influence of loading history, concrete tensile strength, and the quantity of longitudinal reinforcement on the performance of real RC structures. Consideration of these issues in future research on the seismic performance of RC might improve the current confidence levels in newly designed conventional RC structures.
\end{abstract}

\section{INTRODUCTION}

The current understanding of the seismic performance of structural components is largely based on the outcomes and developments of previous research by methods of experimental testing and, in more recent times, numerical modelling techniques. As damaging earthquakes occur relatively infrequently, the information gained from examining the effects of damaging earthquakes provides a rare opportunity to assess whether the previous "laboratory-based understanding" provides a reasonable comparison to field observations.

The performance of some reinforced concrete (RC) buildings in the 2010-2011 Canterbury earthquake sequence was somewhat unexpected compared to previous structural tests performed in laboratories using typical seismic experimentation procedures (which is elaborated in detail later). Such observations have highlighted the need to reconsider the way in which structural tests are undertaken to make them representative of how 'real' RC structures might perform during severe earthquake-induced ground motions. Wider aspects of the performance of RC buildings in the Christchurch CBD have been documented in references 1-4 among others. In particular, the seismic performance of some $\mathrm{RC}$ wall structures was relatively poor.
This paper examines several reasons for the lack of correlation between observations from previous laboratory testing and the damage states sustained by Christchurch buildings during the 2010-2011 Canterbury earthquake sequence. However, the reader should note that this lack of correlation may not necessarily be universal in all scenarios. The 'in-situ field conditions' discussed in this paper could possibly be more relevant to certain structures in Christchurch (or across New Zealand's RC construction) when compared to other international seismic regions built up of other types of RC structures to different construction practices.

Firstly, the typical experimental behaviour of RC structural components is compared to post-earthquake observations in Christchurch buildings. While the first sections of this paper are predominantly focussed on RC wall structures, the intentional of the scope of the paper is to discuss the structural engineering concepts that are relevant for all RC structural members. Although the authors have attempted to distinguish important differences (between RC walls and RC beams, for example) within the paper, the interpretation and application of the technical content of the paper will depend somewhat on the prior knowledge of the reader. Secondly, the effects of the nature and rate of loading, in-place concrete strength, and quantity of longitudinal reinforcement on the behaviour of RC components are discussed. These factors (the typical

${ }^{1}$ Structural Engineer, Holmes Consulting Group, Christchurch (Member) garethm@holmesgroup.com

2 Technical Director, Holmes Consulting Group, and Holcim Adjunct Professor, Department of Civil and Natural Resources Engineering, University of Canterbury, Christchurch (Fellow)

${ }^{3}$ Associate Professor, Department of Civil and Natural Resources Engineering, University of Canterbury, Christchurch (Member). 
"experimental conditions") are further described in an attempt to explain the lack of correlation between laboratory and field observations. Lastly, considerations and challenges for future research and for structural engineering practice are outlined.

A key contribution of this paper is the evaluation of outcomes from a wide ranging search of international literature in order to improve the understanding of the relevant implications for structural engineering practice. Combinations of research and field observations have been included in this paper with the intention of identifying: (i) the inconsistencies in the current body of knowledge, and (ii) the implications for structural engineering practise in New Zealand (and perhaps internationally). Aside from the Canterbury earthquakes, the motivation for presenting this paper arises from multiple recent discussions with practising structural engineers across New Zealand about this particular subject.

\section{EXPERIMENTAL OBSERVATIONS VS. REALITY}

\section{Typical Structural Behaviour and Spread of Plasticity Observed in Experimental Testing}

Laboratory testing of RC components subjected to quasi-static loading protocols has historically exhibited the formation of a ductile plastic hinge zone (PHZ) adjacent to the fixed end region. Countless tests have shown the formation of diagonal flexure-shear crack patterns, examples of which are shown in Figure 1(a) for a RC beam and in Figure 1(b) for a RC wall. These fanned crack patterns progressively develop during simulated seismic loading and lead to the gradual spread of inelastic steel strains (i.e. the "spread of plasticity") from the critical section of maximum bending moment further into the component. Differences in the tensile strain capacity of the

(a)

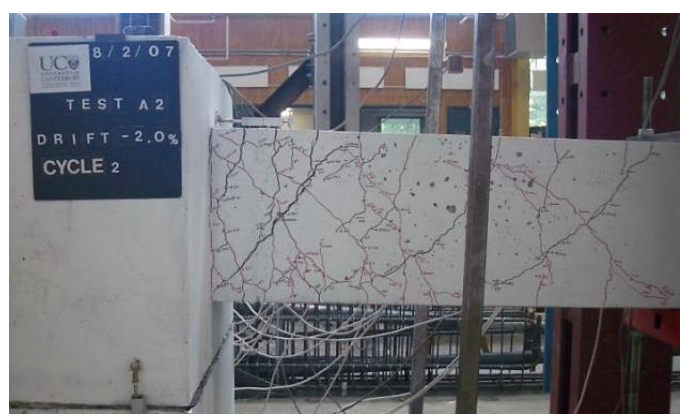

reinforcing steel and concrete mean that some bond slip must occur to accommodate this strain incompatibility. In general, this type of behaviour lengthens the PHZ, which is a significant requirement of ductile RC structure components that are designed to sustain multiple cycles of inelastic deformation. The longitudinal reinforcement will yield over a length equal to a combination of three segments due to: (i) yield penetration into anchorage zones; (ii) the relative increase in the maximum bending moment above the first yield moment due to strain hardening, and; (iii) extension due to tension shift due to diagonal flexure shear cracking 5 .

The "effective plastic hinge length", denoted $L_{p}$ in Figure 1(c), is the length over which plastic curvature is assumed to be uniform for analytical purposes. $L_{p}$ is strongly dependent on the slope of the bending moment (magnitude of shear) at the critical section, moment-shear $M / V$ ratio, quantity of transverse reinforcement, and magnitude of axial load. The length of yielding, $L_{y}$, is referred to as the length of ductile detailing in the New Zealand concrete structures standard 6 (referred to herein as NZS3101:2006), and is schematically shown on Figure 1(c) to be considerably longer than $L_{p}$. The extension of yielding along the member (the "spread of plasticity") also depends on the formation and spacing of flexural cracks, which is dependent on member geometry, tension force in the longitudinal reinforcement, and tensile strength of the surrounding concrete. The size of the flexural tension force is influenced by the quantity and the stress-strain relationship of the longitudinal reinforcement, particularly the maximum strain that can be sustained and the amount of strain hardening. If secondary cracks cannot form between primary cracks, very high reinforcement strains are induced and limited ductility can be sustained before the reinforcement fails 4 .

(b)

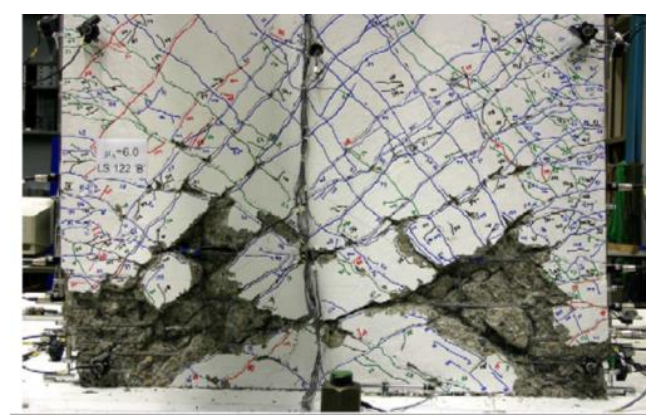

Note -

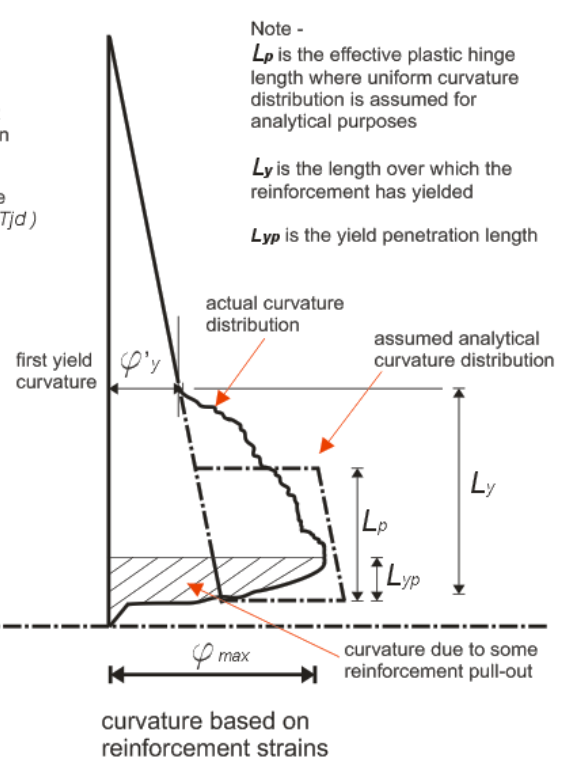

Figure 1: Distributed flexure-shear cracks observed in experimental testing: (a) RC beams tested in 7; (b) a U-shaped RC wall with boundary elements tested by Beyer et al. 8. (c) Expected deformations in the PHZ of monolithic RC walls with distributed flexure-shear cracking. 

The spread of plasticity in real RC structures under seismic actions has long been expected to be consistent with experimental observations from laboratories around the world (e.g. Figure 1). Many of the assumptions for structural behaviour used in practice are based on the outcomes of the experimental studies described in the literature, such as Priestley and Park's (1984) extensive research on the seismic performance of RC bridge columns 9. For the purpose of preliminary design stages, or when using seismic assessment methods such as 10 , the value of $L_{p}$ is one of several simplifying assumptions made in lumped plasticity modelling approaches. This allows for an estimation of the overall structural ductility factor that reduces the force demands on a particular structural system. However, the structural ductility factor does not give a reliable indication of the deformation sustained in a potential PHZ 5,11.

NZS3101:2006 6 considers the value of $L_{p}$ for the purposes of assessing section curvatures and determining the level of detailing required for potential PHZs. For RC beams, columns and walls, $L_{p}$ is coupled with material strain limits (considered by maximum allowable curvatures stated in Table 2.4 of NZS3101:2006). Fenwick and Dhakal 5 used previous experimental data on RC beams, columns and walls to determine the allowable curvature values that corresponded to initially assumed values for $L_{p}$. For reversing plastic hinges in beams, columns or walls, $L_{p}$ is taken as the smaller of half the section depth $0.5 h$ or 0.2 times the $M / V$ ratio, but not less than one quarter the section depth. In both research and practice there has long been a consistent agreement that using $L_{p}=0.5 \mathrm{~h}$ is a reasonable approximation 12. In NZS3101:2006, any error in the assumed values of $L_{p}$ is removed when used with the corresponding material strain limits.

Suggestions for calculating $L_{p}$ by Priestley et al. 13 have been widely adopted in both research and in practice. Equation 1 is described as an "accurate" estimate compared to $\quad L_{p}=$ $0.5 h$, albeit a less conservative estimate with respect to ductility. Equations 1-3 were empirically derived from a database of experimentally measured section and member deformations such that curvature and displacement ductility relationships could be re-arranged and solved for $L_{p}$.

$$
L_{p}=k L_{c}+L_{y p}
$$

where:

$$
\begin{gathered}
k=0.2\left(\frac{f_{u}}{f_{y}}-1\right) \leq 0.08 \\
L_{y p}=0.022 d_{b} f_{y}
\end{gathered}
$$

where $L_{c}$ is stated as the length from the critical section to the point of inflexion, $f_{u}$ and $f_{y}$ are the ultimate tensile strength and yield strength of the longitudinal reinforcement, $d_{b}$ is the diameter of the longitudinal reinforcement, and $L_{y p}$ is the yield penetration length. The factor $k$ considers the slope of the bending moment at the critical section and hence the extension of yielding along the member due to strain hardening of the reinforcement, which appears reasonable.

In practice, the term $L_{c}$ in Equation 1 is somewhat misleading for cantilevered walls in multi-storey buildings for two reasons. Firstly, simplified relationships between curvature and displacement ductility are based on an example of a cantilevered column with a point load applied at the top such that the column height is directly equal to $L_{c}$ and the $M / V$ ratio at the wall base (as shown in the central image of Figure 1). Secondly, some previous tests of RC walls used a concentrated load at the top of the scale-reduced specimens (again the component height is equal to the $M / V$ ratio) and $L_{p}$ was often expressed as a percentage of the specimen wall height. However, the force distribution for structural walls in real multi-storey buildings means the wall height is an inappropriate parameter to relate to the effective plastic hinge length. The moment diagram in Figure 1(c) labels the $M / V$ ratio as the appropriate length along the member that influences the spread of plasticity. This difference is recognised in 13 where $L_{p}$ for cantilevered RC walls is estimated by Equation 4 . The length, $L_{c}$, from Equation 1 is replaced by $H_{E}$, an effective height that should represent the $\mathrm{M} / \mathrm{V}$ ratio for a structural wall.

$$
L_{p}=k H_{E}+0.1 l_{w}+L_{y p}
$$

Equation 4 also includes an additional term of $10 \%$ of the section depth, $0.1 l_{w}$, to account for greater tension shift occurring in walls compared to that in beams. For cracked RC components with combined flexure and axial loading, the trajectory of the internal compressive stress resultant becomes inclined towards the compression zone. As a result, there is a shear force component that is resisted through the concrete and therefore reduces the shear force $V_{s}$ that is resisted by the transverse reinforcement (stirrups in beams/columns or horizontal reinforcement in walls). Considering the moment equilibrium for a free body of a diagonally cracked RC component, $V_{s}$ provides some moment resistance. However, as the axial loading increases and $V_{s}$ reduces, the length of tension shift will increase. This influence of axial loading generally means the length of yielding will be longer in columns and walls than in beams.

Priestley and Park 9 identified that some degree of bond deterioration will increase the length of the reinforcement that will yield as inelastic tensile strains penetrate some distance into the anchorage zones of the longitudinal reinforcement (e.g. beam-column joints and wall footings). As the anchorage zones of RC components are not fully rigid, the relative slip between the reinforcement and concrete (known as bond slip) near the critical section will contribute to the total inelastic deformation. The extent of yield penetration into, or through, a joint will depend on many factors, of which the number and amplitude of inelastic loading cycles will significantly influence bond deterioration

There are many factors that influence the effective plastic hinge length that are not considered in Equations 1 to 4 which have been expressed in this form for ease and simplicity in design practice. Equation 3 suggests $L_{y p}$ depends on the yield strength and nominal diameter of the reinforcement. It was previously suggested that the reinforcement may be expected to yield over a length of 6 times the bar diameter, i.e. $6 d_{b} 9$. More recently, Equation 3 suggests $L_{y p}$ is equal to $6.6 d_{b}$ for Grade 300 reinforcement and $11 d_{b}$ for Grade 500 reinforcement. The following section of this paper explains the significance of the true yield penetration length when this length of the reinforcement becomes the only available source of plastic deformation for components without extensive cracking and spread of plasticity along the member. Some previous structural tests on beam specimens used additional bars welded to the reinforcement passing through the anchorage zones in order to limit the extent of yield penetration $5,7,14$. This technique has been employed to reduce the deformations attributed to anchorage slip to give conservative values of the plastic curvatures that are measured.

The simplified form of Equation 3 suggests that $L_{y p}$ will be the same for identical reinforcement properties, irrespective of the mechanical properties of the surrounding concrete. Without presenting a detailed discussion of the mechanism of bond in this paper, it is widely accepted that the strength of concrete (particularly the tensile strength) significantly influences the relationship between bond stress and bond slip 15. In recent decades there have been significant research developments in understanding bond behaviour at inelastic reinforcement strains 16-21. The literature suggests that the 
extent of yield penetration may be greater for lower grades of steel due to a greater reduction in bar diameter at large inelastic strain demands (known as the Poisson effect). The amount of relative bond slip near the crack plane will depend on the yield stress of the reinforcement if the strain demand is low. In contrast, if the strain demand is large then the local bond slip will depend on the length of the yield plateau and the strain hardening behaviour.

In both research and in practice, the understanding of the structural behaviour of RC and published empirical expressions such as Equations 1-4 have largely emerged from research outcomes of laboratory-based experimental testing. Typical "experimental conditions" that may influence the structural behaviour include: (i) inelastic deformations measured during the application of a gradually increasing symmetric quasi-static loading protocol shown in Figure 4(a); (ii) test specimens containing relatively young concrete with compressive strengths ranging between $25-40 \mathrm{MPa}$, and; (iii) the use of moderate to high proportions of longitudinal reinforcement where there was no restriction of progressive cracking along the member.

\section{Observed Performance of Real RC Structures}

Damage observations in the Christchurch CBD during the 2010-2011 Canterbury earthquake sequence demonstrated that some conventional RC wall structures developed regions of concentrated inelastic deformations that were markedly different than the distributed PHZs observed in experimental tests. When inelastic reinforcement strains are concentrated at a small number of large cracks the available ductility of the component is significantly reduced.

Figure 2(d) illustrates the deformations in lightly reinforced walls developing a "single-crack-PHZ". The formation of the primary crack at the critical section reduces the tensile stress in the concrete over a reasonable height up the wall. The location where the next primary crack might form, denoted $L_{p c}$ on Figure 2(d), is theoretically between one and two times the distance from the extreme tension fibre to the neutral axis at the initial crack 22-23. At a distance of $L_{p c}$ from the critical section, the flexural tension force might be less than the tensile strength of the surrounding concrete and the next potential crack cannot form. As a result, significant strain hardening of the reinforcement must occur at the critical section to increase the flexural tension force along the member and increase the possibility of developing the next potential primary crack.

This concentration of reinforcement strains significantly reduces the effective plastic hinge length that may be adopted for analytical purposes. Some buildings in this category exhibited much less available ductility than previously anticipated. Due to the lack of cracking along the member, the elongation of the reinforcing steel will depend on the magnitude of the steel strain and the true extent of yield penetration back into the surrounding concrete. $\mathrm{RC}$ walls with a single-crack-PHZ require significant bond deterioration adjacent to the crack in order to withstand the overall lateral deflection demands of the building. However, in cases where the vertical reinforcement had good bond conditions, and high bond stresses could be sustained, the extent of yield penetration might have been less than previous empirical suggestions such as Equation 3. The major consequence of this behaviour is the high strain concentrations and possible brittle failure of the vertical reinforcement. An example of this behaviour includes the 11 storey Gallery Apartments building shown in Figure 2(a)-(c).

The vertical reinforcement in the critical wall of the Gallery Apartments contained two layers of HD12's (Grade 500, $12 \mathrm{~mm}$ bars) spaced at $420 \mathrm{~mm}$. The rectangular section was $4300 \mathrm{~mm}$ in length and $325 \mathrm{~mm}$ thick. For the as-built details the longitudinal reinforcement ratio was $0.16 \%$, whilst the minimum required by NZS3101:2006 6 for this section is $0.27 \%$. Further discussion of the quantity of longitudinal reinforcement is presented in a later section of this paper.

Initial assessments suggested the crack width during seismic excitation would have been in the range of $35 \mathrm{~mm} \mathrm{2}$. At first visual inspection, the crack appeared to be relatively narrow and the damage to the building was not an obvious concern. However, in reality, the crack opened to a significant width as the wall deflected during seismic excitation, but closed as the wall re-centred itself under gravity load. Figure 2(b) shows the Urban Search and Rescue team from New South Wales who found several reinforcing bars had fractured along the length of the wall, as shown in Figure 2(c). The building's overall damage state may be described as being at near collapse. A potentially catastrophic failure might have been observed for a

slightly longer duration of severe ground shaking. This example also highlights the care required in assessing a damaged building of this type.

The Pyne Gould Corporation (PGC) building is an example of a lightly reinforced wall building that catastrophically collapsed during the 22 February 2011 Christchurch earthquake, in which 18 people lost their lives (CERC, 2012). Due to the building being designed in the mid-1960s, it was originally categorised as a non-ductile RC structure. The postulated critical wall was $203 \mathrm{~mm}$ thick and contained a single layer of $16 \mathrm{~mm}$ vertical reinforcement spaced at $380 \mathrm{~mm}$. There was a large cover concrete thickness of nearly $6 d_{b}$ and the vertical reinforcement had good bond conditions which might have limited the extent of yield penetration that occurred. Elongation of the vertical reinforcement was limited to a short length and bar fracture is postulated to have occurred in the sequence of collapse. Further discussion of the building's seismic performance and potential collapse scenario is presented in the CERC report 3 .

The authors are aware of other lightly reinforced wall structures in Christchurch that formed concentrated regions of inelastic deformation during the Canterbury earthquake sequence 1,3 . Practicing structural engineers throughout New Zealand will encounter existing buildings with RC walls designed to earlier standards (NZS3101:1995, Error! Reference source not found.) that have a similar vulnerability. This does not mean to say, however, that similar issues may not exist for other structural members such as RC columns. Another reason for single-crack opening may have been the common construction practice that used "cold-joints" and insufficient development lengths for lapped splice regions at the wall base. 
(a)

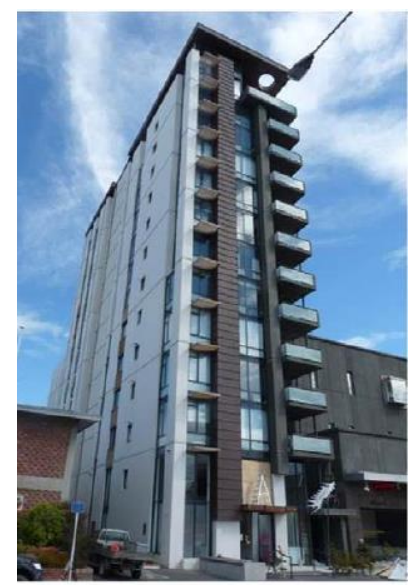

(b)

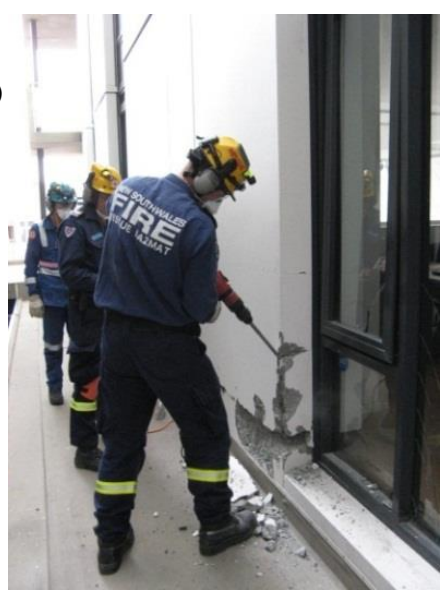

(c)

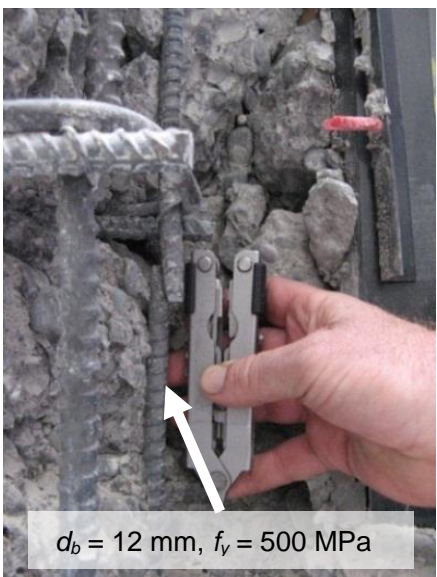

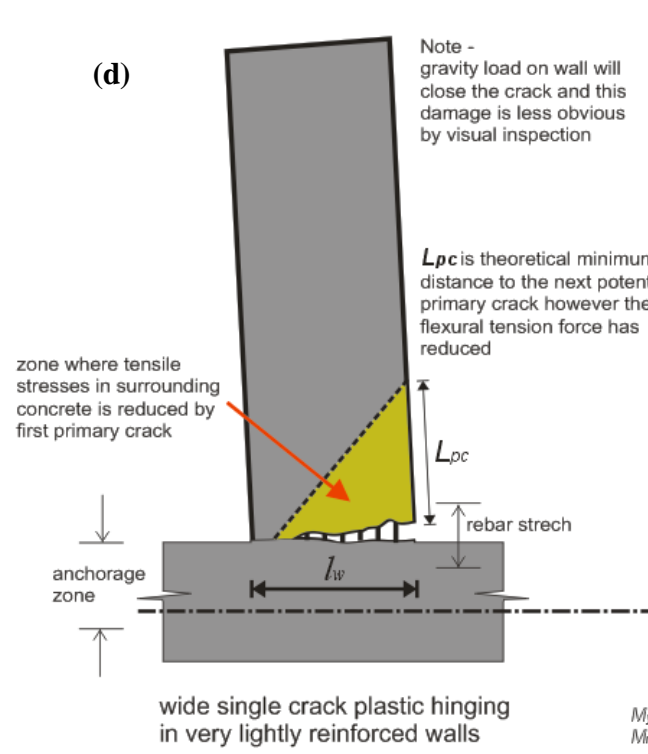



$M y 1$ is moment at first yield
$M m$ ax is maximum moment at critical section

Figure 2: Illustration of frequently observed PHZ behaviour in the Canterbury earthquakes: (a) The 11 storey Gallery Apartments RC wall building; (b) Urban Search and rescue (USAR) team removing cover concrete; (c) fractured vertical reinforcement [2]; and (d) schematic illustration of deformations in the case of a single-crack-PHZ for monolithic RC walls.

Some post-earthquake reports 24,26 describe examples of beams that formed apparent single-crack-PHZs as shown in Figure 3. The CERC report 3 discusses beams containing sufficient longitudinal reinforcement such that secondary cracks were able to form; however crack widths were generally very narrow (less than $0.05 \mathrm{~mm}$ ) and were not clearly visible. Bar yielding at secondary cracks can only occur if there is significant strain hardening at the nearby primary crack, meaning that appreciable strains must be induced and primary cracks need to be sufficiently wide (up to about $5 \mathrm{~mm}$ ). This kind of behaviour is not overly concerning as it is consistent with what has been observed in experimental tests on beams at a displacement ductility in the range of 2-3. Secondary cracks are somewhat easier to inspect during laboratory testing and all cracks are clearly marked on the test specimens during static loading.

In summary, damage states observed in post-earthquake field reconnaissance of some RC walls were arguably not consistent with the spread of plasticity observed in previous experimental testing in the laboratory, thus highlighting the need to review and calibrate the current laboratory-based understanding for the behaviour of RC structures. Some of the typical seismic experimentation procedures and known laboratory conditions have potentially influenced the structural behaviour and longheld assumptions for RC that are used in research and in practice. Clear differences between conventional laboratory and field conditions include: (i) the type of dynamic loading from the 22 February 2011 Christchurch earthquake was significantly different to the typical quasi-static loading protocols often used in laboratory testing, and; (ii) real structures had significantly less cracking of the concrete, and hence the ductility of the reinforcement was not well utilised compared to laboratory test specimens. The following section discusses the influence of loading history on the behaviour of $\mathrm{RC}$ and the use of quasi-static testing. Later sections of this paper discuss the significance of concrete tensile strength and quantity of longitudinal reinforcement on the restricted cracking and limited spread of plasticity in real structures.

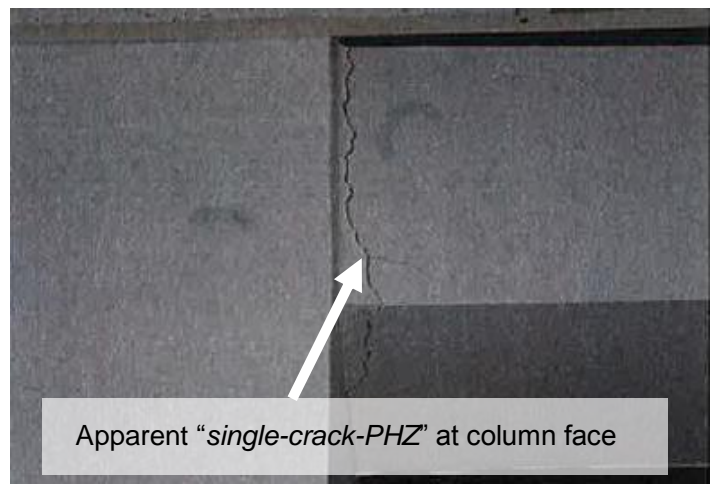

Figure 3: One example of localised damage observed in a RC frame building [24]. 


\section{INFLUENCE OF LOADING HISTORY}

\section{Underlying Issues with Seismic Experimentation}

For many laboratories conducting seismic experimentation, shake-table or other real-time dynamic testing is constrained by resources and practicality (such as cost, available equipment, required computer software, support of laboratory technicians). To avoid these constraints, quasi-static cyclic loading is the most widely implemented method for structural tests 27. The results of quasi-static testing are assumed to provide a conservative lower bound for member strength capacity; however the same cannot be said for ductility and energy dissipation. The technical disadvantages are that quasistatic testing cannot consider: (i) the influence of the loading rate on governing failure mode and; (ii) variations in momentshear ratios and axial load that largely influence the deformation and strength capacities. The deformation and strength capacity depends on the cumulative damage due to the path-dependent behaviour of RC 28. The authors noted that more recent experimental tests on external RC beamcolumn joints have included axial load variations in quasistatic testing 29, (among others at the University of Canterbury and University of Pavia in the past decade).

For components within a real structure, the amplitude, frequency and number of loading cycles (i.e. seismic demands) due to ground motion excitation depends on:

- The influence of earthquake source rupture, seismic wave propagation and local site response on the features of ground motion intensity measures: amplitude frequency content and duration.

- The configuration and relative strength of the component within the global system.

- Dynamic system properties such as stiffness, natural modes of vibration and inelastic response (ductility and hysteretic energy dissipation).

For several decades researchers have been aware of the need for generalized experimental loading protocols to reliably evaluate and compare the performance characteristics of structural components 30 . More recently, the popular notion of performance-based design has highlighted the importance of performance indicators such as deformation capacity to be used in design procedures and standards. Loading protocols are recognised as a source of epistemic uncertainty associated with evaluating performance indicators (or damage states) in the development of component fragility functions used for performance-based seismic assessment 31 .

\section{Quasi-Static Loading Protocols}

Liddel et al. 14 found differences in the ductility capacity of RC components when subjected to varying quasi-static loading protocols used at different international research institutions. Loading protocols need to be reflective of the experimental objectives which may vary from determining potential failure modes to assessing the drift sensitivity of nonstructural elements. FEMA-461 32 suggests quasi-static loading protocols should be generalized such that the sequence of displacement cycles are in order of increasing magnitude to ensure that component performance is not unique for specific ground motions and configurations, but for a range of potential displacement histories. Figure 4(a) and (b) shows typical loading protocols that have been widely used in experimental quasi-static testing of $\mathrm{RC}$ components that undergo strength and stiffness degradation in a gradual manner. Under this type of gradually increasing loading, crack propagation is more extensive which enables greater spread of plasticity and therefore significant levels of deformation capacity and hysteretic energy dissipation, while premature failure modes such as bar buckling or bar fracture are mitigated. It should be recognised that empirically-derived expressions for the effective plastic hinge length and estimates for the yield penetration length which are widely used in practice are based on outcomes of quasi-static testing using loading protocols such as that shown in Figure 4(a).

In contrast to typical quasi-static loading protocols, severe near-source ground motions from damaging earthquakes, such as the 1971 San Fernando (US), 1994 Northridge (US), 1995 Kobe (Japan) and 2011 Christchurch (NZ), can produce initially large amplitude, high frequency, and partially reversing loading histories without a number of initial small amplitude or gradually increasing loading cycles. FEMA-461 (2007) ignores the influence of near-source ground motions on the basis that these motions generate fewer response cycles and therefore are not likely to control the number and relative amplitudes of the loading excursions in a loading history. Krawinkler 28 discussed various loading protocols used for multi-institutional testing programmes and standards, such as those shown in Figure 5(a) and (b) for steel and timber structures, respectively, with attempt to assess the seismic performance when subjected to near-source ground motions with forward directivity. However, no specific loading protocol for RC structures has been commonly discussed in the literature.

\section{Loading Rate}

Despite the awareness that near-source events result in dynamic large amplitude ground motions, there are few consistent conclusions in the literature for the influence of loading rate on the seismic performance of RC components. Quasi-static loading potentially mitigates brittle failure modes that are otherwise realistic for real RC structures subjected to realistic seismic actions. Vos and Reindhardt 33 found that deformed reinforcing bars have greater bond resistance when subject to faster loading rates and this enhancement for bond strength was more pronounced for lower quality concrete. As the concrete matrix becomes more uniform in high quality concrete, the relative micro-crack propagation is limited and less concrete degradation occurs. Chung and Shah 34 investigated the effect of loading rate on small scale anchorage-bond and beam-column joint specimens and observed fracture of the reinforcement when subjected to faster loading rates.

References 35 and 36 performed structural tests on RC components with input shake-table motions representative of "near-source" earthquakes (containing large asymmetric pulses) and "far-field" earthquakes. Both studies tested RC bridge columns containing relatively high quantities of longitudinal reinforcement (between 2.0-3.6\%). There was no evidence of concentrated inelastic deformations that the ductility capacity of components with moderate or high reinforcement content is unlikely to be influenced by loading rates, which is in agreement with 3; however further investigations should be carried out for lightly reinforced components.

In the interest of producing realistic experimental outcomes, laboratory facilities within New Zealand could benefit from upgrading so that shake-table testing can be performed at a more appropriate geometric scale without being constrained by the speed at which loading is applied. However, conducting large scale experimental tests continues to be a relatively expensive task. Another approach might consider a combination of smaller experimental studies and detailed analytical modelling. The dynamic material response of reinforcing steel and concrete could be studied in experimental tests to determine the inputs in analytical models for predicting the behaviour of RC structures. Alternatively, the influence of dynamic loading rates could be experimentally investigated at international research institutions with superior 
laboratory facilities 37 . In a current study 38 there is some early analytical evidence for the influence of loading rate on

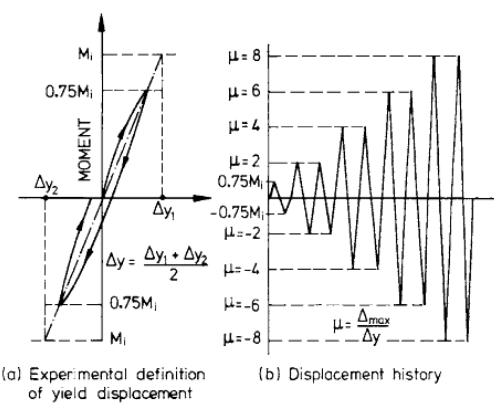

(a) Priestley and Park 9
RC structural models (with 'as-built' details).

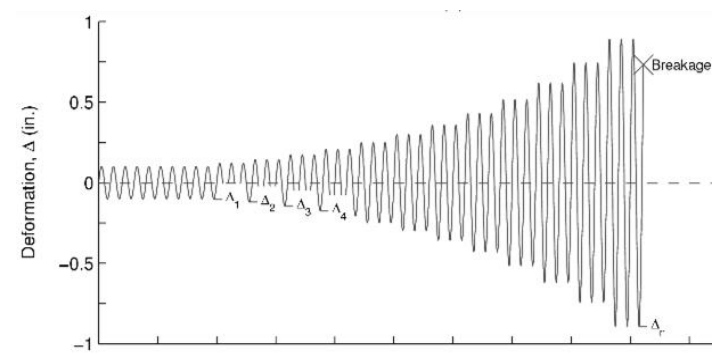

(b) Loading protocol stated in FEMA-461 32

Figure 4: Typical examples of loading protocols for quasi-static cyclic testing.

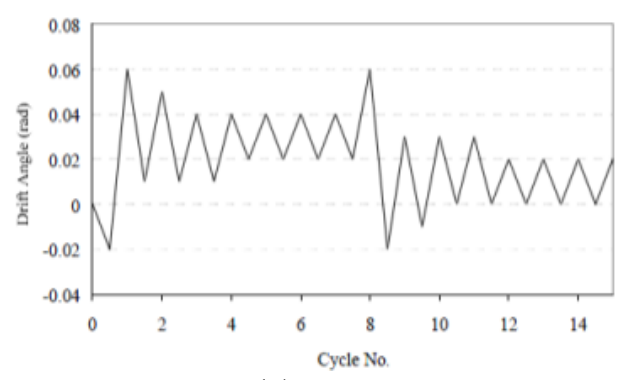

(a)

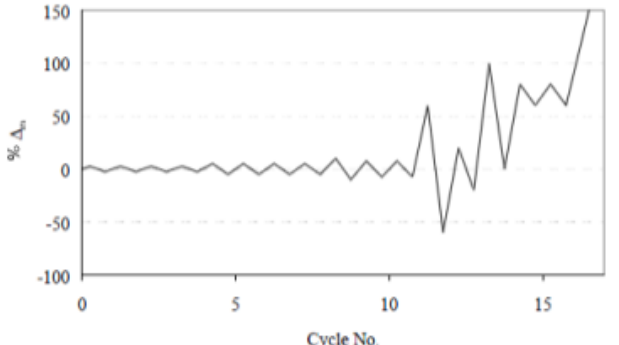

(b)

Figure 5: "Near-source" loading protocols for (a) structural steel components, and (b) timber components 28.

\section{IN-PLACE CONCRETE STRENGTH}

\section{Materials Testing}

Damage observations and materials testing from Christchurch CBD buildings indicate that the strength of concrete surrounding the reinforcement was notably higher than that specified in design. The CERC report 3 describes the unexpected performance of several RC structures to be largely due to the reoccurring issue of higher-than-expected concrete strength. This section discusses some evidence of higher than expected concrete strength, the concrete tensile strength, some apparent factors that might enhance the concrete strength, and relevant considerations for future research.

From material testing of samples extracted from a number of Christchurch CBD buildings it was found that the in-place strength was significantly higher than expected 39. The Gallery Apartments building (Figure 2) is an example where the specified 28 day compressive strength, $f_{c, 28 \text {-days, was }}^{\prime}$ $30 \mathrm{MPa}$, however, Holmes Solutions 39 reported that the cylinder compressive strength of two cores extracted from the critical walls were $46.5 \mathrm{MPa}$ and $56.0 \mathrm{MPa}$, and nondestructive Schmidt hammer testing indicated a compressive strength in the range of 54-70 MPa. Two split cylinder tests measured the "indirect tensile strength" of $2.4 \mathrm{MPa}$ and 3.4 MPa.

\section{Concrete Tensile Strength}

In practice the tensile strength of concrete is typically given greatest consideration at the serviceability limit state. Design codes typically state lower characteristic values for the tensile strength to provide some conservatism in calculating the strength capacity and deflection-induced cracking under serviceability loads. However, the earlier sections of this paper highlighted that the performance of some structures at the Ultimate Limit State (ULS) may be significantly influenced by the concrete tensile strength. It is widely accepted that high strength contributions from the concrete between the cracks (often referred to as "tension stiffening") will result in PHZs having a much lower rotation capacity. The tensile strength is also known to have a strong influence on bond behaviour [14]. At ULS the concrete tensile strength may be a critical factor that restricts the available ductility of the reinforcement that is utilized due to: (i) secondary cracks are unable to develop; and (ii) limited bond deterioration near single-crack-PHZs. These issues suggest the need to carefully consider what the probable values of the tensile strength might be when a lightly reinforced structure is being assessed.

Figure 6(a) gives an indication of the relationship between the mean compressive strength and "direct tensile strength" $f_{c t}$ by using the expressions shown in Equations 5 and 6. These expressions are from the Model Code 201020 and a proposed amendment to NZS3101:2006 6, respectively. Figure 6 also shows upper and lower characteristic values which are taken as 1.32 and 0.68 times $f_{c t}$ in the Model Code 2010 (and similarly in the commentary section of NZS3101:2006). The scatter in the concrete tensile strength represents the influence of several factors, including: the extent of cement hydration; member geometry and differential shrinkage; the proportion, size and angularity of course aggregate; and segregation of constituent materials in casting. For higher grades of concrete ( $f_{c}^{\prime}$ exceeding say 60-70 MPa) the relationship between direct tensile strength and compressive strength will vary from that suggested in Equations 5 and 6.

$$
\begin{gathered}
f_{c t}=0.3\left(f^{\prime}{ }_{c}\right)^{\frac{2}{3}} \\
f_{c t}=0.55\left(f^{\prime}{ }_{c}\right)^{\frac{1}{2}}
\end{gathered}
$$

The aforementioned observations in some Christchurch buildings indicate that an upper characteristic value should be taken as the effective tensile strength for the purposes of assessing whether secondary crack formation can occur. Figure 6(b) qualitatively shows the relative differences between the three measurements of concrete tensile strength, in which it can be seen that: 
- The indirect tensile strength is determined from splitting (or "Brazilian") tests which are easily performed on small cylinder specimens. There is typically a large amount of scatter in the results from performing a small number of splitting tests.

- The direct tensile strength, or uniaxial tensile strength, is seldom measured due to the difficulty in test set up and loading concrete specimens in direct uniaxial tension 40. In most design codes the direct tensile strength is taken as $90 \%$ of the indirect tensile strength.

- The flexural tensile strength, or modulus of rupture (MoR), may be determined relatively easily from third-point loading of plain concrete prisms. These tests are not carried out on samples from buildings however as samples are typically extracted in the form of cylinders (hence splitting tests are most commonly used).

The relative difference between flexural and direct tensile strengths of plain concrete arises due to a combination of

(a)

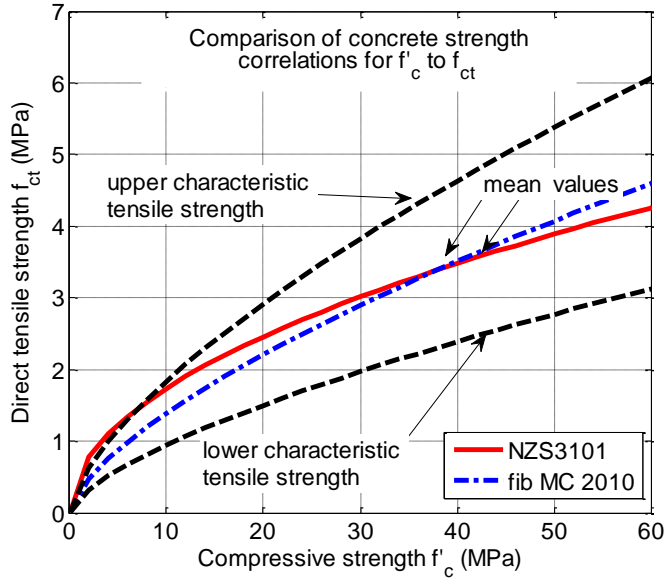

material and geometric non-linearity (Gopalaratnam and Shah, 1985). This difference is described in the commentary section of NZS3101:2006 and recommended multipliers for this scale effect (Table C5.1, NZS3101:2006) are approximately identical to those determined using Equation 7 and 8 from the Model Code 2010 (fib, 2012) based on fracture mechanics for concrete structures.

$$
\begin{gathered}
\alpha_{f}=\frac{0.06 h^{0.7}}{1+0.06 h^{0.7}} \\
f_{c t, f l}=\frac{f_{c t}}{\alpha_{f}}
\end{gathered}
$$

where $h$ is the section depth and $f_{c t}$ is the direct tensile strength which is often estimated from Equation 5 or from splitting tests.

\section{Figure 6: (a) Examples of some known correlations between the compressive and tensile strength of concrete; and (b) qualitative representation of the relative flexural, indirect and direct tensile strengths of concrete}

Assessments of secondary crack formation for the critical wall in the Gallery Apartments building are presented in 41-43. using analytical modelling techniques. Henry 41 estimated the flexural tensile strength using Equation 8 , where $f_{c t}$ was taken from Equation 5 based on the $f_{c}^{\prime}$ values reported by 39. For typical wall sections, the difference between direct and flexural tensile strength values is minimal due to the relatively large section depth. Input values for the mean and upper characteristic flexural tensile strength of the concrete were taken as $4.3 \mathrm{MPa}$ and 5.6 $\mathrm{MPa}$, respectively. These values were determined using the Model Code 2010 expressions 20 and both were considered as permutations in the analysis. The next section of this paper briefly discusses the limited crack formation observed in the results from 41 and 42.

Identifying that the compressive strength was appreciably higher than specified design values highlights the benefit in obtaining results from materials testing. Other approaches to assessing RC structures might depend on values of the compressive strength determined by non-destructive material testing. Variability in the results from all techniques for concrete materials testing should be considered appropriately, and potential reasons for the measured strength exceeding the specified design values are discussed in the following section. (b)



\section{Sources of Apparent Strength Enhancement}

For all concrete structures, the in-place strength will vary between different components due to the influence of casting direction and size effects. Construction methods used for placement, compaction and vibration (and potential revibration) of concrete also have some influence on strength. The direction of casting relative to the orientation of the structural component will influence the concrete's mechanical properties due to water gain 44. Due to segregation of the mix materials, concrete at the bottom of specimens is typically of higher strength than concrete in the middle, with lower strength concrete at the top. This notion suggests the concrete strength would have been higher at the base of some cast insitu wall structures (cast in the vertical direction) where limited cracking was observed. For the future design of lightly reinforced components, 2 describes the need to consider concrete strength enhancements due to the following factors:

- Ready-mix suppliers targeting higher strength for quality assurance of the delivered concrete product.

- The ageing/maturing process resulting in a timestrength development.

- Dynamic strength enhancements when subjected to rapid loading rates (the implications of which were alluded to in an earlier section of this paper).

- Precast fabricators using high strength and high early strength mixes to meet specification quickly to ensure speed of production. 
Some flowable self-compacting concrete (SCC) mixes can result in high strengths that have not been anticipated by the design engineer. SESOC 45 describes an example of a RC panel with a specified $f_{c, 28 \text { days }}^{\prime}$ of $40 \mathrm{MPa}$, yet a selfcompacting mix had a 7 day strength of $90 \mathrm{MPa}$. In another case example, a relatively modern $\mathrm{RC}$ building had precast wall panels with a 28 day strength of approximately $90 \mathrm{MPa}$ such that the wall's internal actions were higher than might have been anticipated in design and subsequently contributed to failure of the foundations 26 .

Reference 46 contains information on recent quasi-static cyclic testing of four interior beam-column joint specimens containing high-strength SCC. At the time of testing, $f_{c}^{\prime}$ ranged between 100 and $120 \mathrm{MPa}$ and the average indirect tensile strength was 7.2 MPa. The ratio of the longitudinal beam reinforcement was identical for all test specimens at $1.1 \%$. Despite the high tensile strength of the concrete, the quantity of reinforcement and hence the tension force in the reinforcement was sufficient to progressively crack the concrete. Observations from these tests highlight that there were no restrictions on the formation of secondary cracking and thus the desired "spreading-PHZs" were able to form adjacent to the column faces. This finding also agrees with recent analytical evidence presented in 38 .

\section{"Laboratory" Concrete vs. "Real" In-place Concrete}

Some design expressions that are influenced by concrete strength (such as quantities of minimum reinforcement and development lengths) are derived from experimental work. While such expressions may account for some scatter by carrying out an appropriate number of tests, there may be some debate that the concrete used in the laboratory conditions may not reliably represent of the concrete used in real construction.

To reduce the time in undertaking experimental studies, concrete samples of RC specimens are typically tested at the milestone of 28 days after casting when the concrete is relatively young. The tensile strength in younger test specimens may be appreciably less than the strength of inplace concrete in an existing structure of some age. Some experimental programmes might allow for 90 days of strength development to reduce the variability between specimens that are of slightly different maturity at the time of testing. A search of the literature or further experimental research is needed to investigate the rate at which concrete tensile strength develops. Research institutions and the New Zealand concrete industry should take careful consideration of the mix that is used in specimen construction and the age of concrete at the time of testing.

\section{QUANTITY OF LONGITUDINAL REINFORCEMENT}

To utilize the ductility of the reinforcement at ULS there needs to be sufficient tension force in the longitudinal reinforcement to progressively form cracks along the potential PHZ. The aim of code limitations for the minimum reinforcement quantity, $\rho_{\min }$, is to prevent the formation of a single wide crack once the cracking moment of the section has been exceeded. To ensure a factor of safety against this undesired behaviour, the nominal moment capacity of a section with minimum reinforcement should be approximately 1.5-2.0 times the cracking moment (Paulay and Priestley, 12). Reference 41 further describes the background of the design expressions for the minimum reinforcement in RC beams and walls.
The minimum reinforcement ratio stated in NZS3101:2006 6 for both walls and beams is given by:

$$
\rho_{n} \geq \frac{\sqrt{f_{c}^{\prime}}}{4 f_{y}}
$$

where $f_{c}^{\prime}$ is the specified 28 day strength (MPa) and $\rho_{n}$ is the calculated total area of longitudinal reinforcement as a ratio of the area of the concrete section dimensions using the width of the web and the section depth, $b_{w} d$.

While Equation 9 appears to be identical for walls and beams, there are a number of differences between each component that reduces the safety margin between the nominal and cracking moment capacity for wall sections 41 . For example, the expression for walls is the total quantity of vertical reinforcement that is distributed through the section, while for beams the expression represents only the quantity of reinforcement that is in tension, which is typically lumped in the flange regions.

An important difference between RC test specimens and components in real structures is that test specimens will typically contain moderate and high quantities of reinforcement. To minimize concrete volumes and specimen weight, the geometry of test specimens is often reduced in scale such that test specimens contain a higher proportion of reinforcement compared to real structures. Reducing the proportion of longitudinal reinforcement with specimen geometry is uncommon. This is based on a misconception that if there is good structural behaviour for high values of $\rho_{n}$ then there should also be good behaviour at lower values. It should be noted that modern experimental programmes are expected to follow similar criteria where equal stresses and constant density of the reinforcement are adopted.

\section{RC Walls}

SESOC 47 responded to the poor performance of lightly reinforced walls in the 2010-2011 Canterbury earthquakes with a proposed design recommendation for the minimum quantity and distribution of reinforcement for walls that are likely to yield. This interim design recommendation offered some improved confidence that newly designed walls can develop the desired flexure-shear crack patterns and achieve ductile behaviour at ULS. Equation 10 shows that the expression from 47 was increased from the NZS3101:2006 6 minimum quantity of vertical reinforcement to account for the higher than expected concrete strength of up to 2.5 times the 28 day specified value:

$$
\rho_{l} \geq \frac{\sqrt{2.5 f_{c}^{\prime}}}{4 f_{y}} \rightarrow \rightarrow \rho_{l} \geq \frac{0.4 \sqrt{f_{c}^{\prime}}}{f_{y}}
$$

Analytical moment-curvature and force-displacement results for the response of the critical wall in the Gallery Apartments building are presented in 41, for two cases: (i) using the asbuilt details with a reinforcement ratio of $0.160 \%$; and (ii) approximately equal to the NZS3101:2006 minimum vertical reinforcement limits of $0.274 \%$.

In the first case, the predicted wall damage was in agreement with the bar fracture that was observed after the 22 February 2011 Christchurch earthquake (previously shown in Figure 2(c)). Figure 7(a) shows fracture of vertical reinforcement occurring at a lateral drift of $0.75 \%$.

The results for the second case were found to be strongly dependent on larger magnitudes of axial loading to avoid sudden losses in strength after cracking and to sustain greater lateral deflections. Although the second case satisfied the NZS3101:2006 minimum reinforcement limit, the effective plastic hinge length was found to be approximately half of the length that is typically assumed in practice 41 . More detailed 
finite element analysis of this case example is presented in 42,48. No analytical evidence was presented for recommendation made by 47 in Equation 10 .

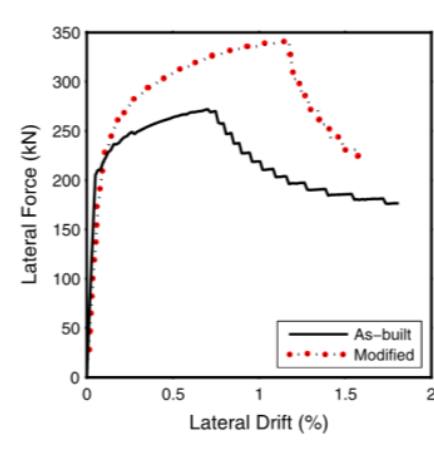

(a)

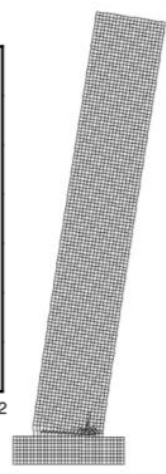

(b)

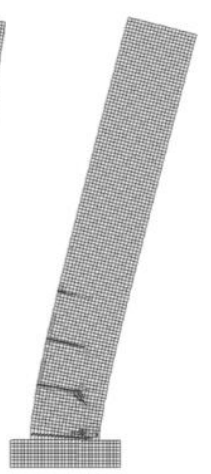

(c)
Figure 7: (a) Analytical predictions for the critical wall section in the Gallery Apartments building for: (b) the asbuilt details, and; (c) the wall containing the NZS3101:2006 minimum vertical reinforcement content [41, 47].

Brief discussion of the distribution of vertical reinforcement in $\mathrm{RC}$ walls is included here for the sake of completeness. The majority of existing wall structures designed according to NZS3101:2006 6 and earlier standards are typically comprised of uniformly distributed vertical reinforcement throughout the section. Following the Canterbury earthquakes, SESOC 47 recommended that modern wall sections should contain greater quantities of reinforcement in the end regions (commonly referred to as boundary elements). The quantity and detailing of web reinforcement is also important for several reasons: (i) to prevent crushing of the concrete in the web; (ii) to prevent the web from forming a small number of wide cracks that may result in potential shear sliding; and (iii) to ensure that severe damage to the web region does not result in excessively large compression stresses and potential buckling of boundary elements 48 .

The design provisions for $\mathrm{RC}$ walls have undergone some revision in Amendment 3 of NZS3101:2006 6 to prevent some of the undesired structural behaviour that has been described in this paper. Changes to the Standard have specified that end zones of walls must have a ratio of vertical reinforcement $\rho_{l, e}$ that is greater than Equation 11, twice the value that was previously considered as the minimum.

$$
\rho_{l, e} \geq \frac{\sqrt{f_{c}^{\prime}}}{2 f_{y}}
$$

The minimum reinforcement in the web region of the wall between recognised end zones must be greater than the ratio stated in Equation 9 and should also be greater than 0.3 times $\rho_{l, e}$. This second limit has been imposed to prevent the issues associated with shear strength and shear deformation as described earlier.

The University of Auckland is continuing to investigate experimentally and analytically the performance of lightly reinforced walls 43 .

\section{RC Frames}

Compared to walls, there are fewer structural and geometric conditions influencing the margin of safety between the nominal moment capacity of a typical beam section and the cracking moment capacity. Despite some apparent cases of "single-crack-PHZs" in RC beams (e.g. Figure 3) this was partly due to misinterpretation of this type of damage observation and is not overly concerning. Thus far, there has been no suggestion of revising the minimum reinforcement for beams, however further experimental investigations may address this issue in more detail.

To give some quantitative indication, a desktop study of the structural drawings for $21 \mathrm{RC}$ frame buildings in the Christchurch CBD (within the post-1977 construction era) was conducted by the authors. The average reinforcement ratio in the ductile regions of beams within the lateral load resisting "seismic frames" was approximately 0.85 and 0.70 percent for top and bottom reinforcement, respectively. The study focused on beam elevations in the lower third of the frame height, though beams located in upper levels of high-rise buildings typically contain a lower proportion of reinforcement. Across New Zealand's existing building stock there will be some RC columns that have been designed to form ductile PHZs. However insufficient vertical reinforcement or changes in cross-sectional dimensions could mean that single-crackPHZs are more likely to form.

\section{CONSIDERATIONS FOR FUTURE RESEARCH AND PRACTICE}

The lack of correlation between the observations from previous structural tests and observations from buildings in Christchurch has highlighted some implications for the design and assessment of conventional RC structures. The following section briefly describes some other issues associated with the structural behaviour that was described earlier.

\section{Flexural Stiffness of RC Components}

One implication related to the limited cracking in some wall structures is that some assumptions for the effective stiffness of RC structures may be inappropriate. Design standards such as NZS3101 6 use multipliers of the gross-section properties that are based on extensive flexural cracking (Table C6.6, NZS3101:2006). Given that the extent of flexural cracking observed in some Christchurch buildings was much less than expected, the fundamental vibration period is likely to be less than what the structure was designed for and consequently the seismic forces may be higher than expected. Fenwick 4 recommends that practicing engineers compare the anticipated stiffness and strength degradation that is associated with the design actions from structural analysis.

\section{Effective Plastic Hinge Length}

Practicing engineers often make some lumped plasticity assumptions in using non-linear modelling techniques to predict the seismic response of a structure for the purposes of detailed seismic and damage assessments or in the design stages. Coupled with the assumed value for the effective flexural stiffness, the assumed length for the effective plastic hinge, $L_{p}$, is also important for structural analysis. Equations 1 and 4 presented some empirical expressions for $L_{p}$ that engineers might adopt as the length over which plastic curvatures are assumed to be uniform. The seismic assessment guidelines in NZSEE 10 state that half the effective section depth may be used for $L_{p}$, or taken "more accurately" and less conservatively by Equation 1 from 13 . While these expressions might be adopted to evaluate the ductility of an existing structure, it is unclear whether the results from analysis are compared against allowable material strains that correspond to performance/damage states. In practice, estimates of ductility derived from nonlinear pushover or seismic response history analysis are commonly presented using other engineering demand parameters such as plastic hinge rotations and/or drift angles. The material strain limits can easily be overlooked if a blind value of $L_{p}$ is assigned inappropriately and ductility may be over-estimated. 
NZS3101:2006 6 assumes $L_{p}$ for the purposes of assessing section curvatures and detailing requirements for potential plastic hinge zones. The value of $L_{p}$ for RC beams, columns and walls is coupled with material strain limits (maximum allowable curvatures stated in Table 2.4 of NZS3101:2006). For reversing plastic hinges in beams, columns or walls the value of the effective plastic hinge length should be taken as the smaller of half the section depth or 0.2 times the $M / V$ ratio, but not less than one quarter the section depth.

Since the Canterbury earthquakes there have been no recommendations to change the value of $L_{p}$ that is used in non-linear modelling techniques. In the interim, $L_{p}$ for lightly reinforced components could be taken as a variable parameter in the analysis input. Evaluations of the available ultimate deformation capacity of a system may input $0.5 h$ as an upper-bound value for $L_{p}$. Considering the kind of damage observations from some buildings in Christchurch, it may be more appropriate to run some permutations in the analysis with a lower-bound input value of $L_{p}$. The lower-bound will predict less available ductility, which may be foreseeable for the response of some RC components containing low quantities of longitudinal reinforcement.

\section{Estimating Ductility in Lightly Reinforced Components in Practice: Example}

There will be cases where structural engineers have carried out post-earthquake seismic response predictions for damaged RC structures that formed single-crack-PHZs, as illustrated in Figure 2(d). In this case, the effective plastic hinge may be restricted to the true length of yield penetration that can occur either side of the primary crack. Based on recent field observations, $L_{y p}$ might be taken as 1 to $2 d_{b}$ to provide a representative lower bound.

An example found in 49 shows how the ductility of a very lightly reinforced component was assessed based on a relatively simple and robust methodology as an alternative approach to that outlined in [12] for the yield penetration in $\mathrm{RC}$ components. In this particular case, a single-crack-PHZ was expected to form at the critical section of $\mathrm{RC}$ dam spillway piers (constructed in the 1960s). The longitudinal reinforcement comprised of $32 \mathrm{~mm}$ deformed reinforcing bars. The cracking moment and first yielding moment for the component was estimated to be $4600 \mathrm{kNm}$ and $2100 \mathrm{kNm}$, respectively, thus leading to concerns about the significance of single-crack-PHZ behaviour on the fatigue behaviour of the reinforcement.

By implementing some existing models found in the literature, Davey and Blaikie 49 considers how the ductility of the component was influenced by: (i) the estimated low-cycle fatigue behaviour of the reinforcing bar; and (ii) the estimated maximum crack width based on an estimated amount of bond deterioration once the deformed bar has yielded. Several assumptions were made in order to use these models from existing literature. Based on some awareness of material properties of reinforcing steel that was available in New Zealand in the $1960 \mathrm{~s}$, a ratio $f_{u} / f_{y}$ of 1.50 was assumed. Materials testing of concrete core samples suggested that $f_{c}^{\prime}$ was $60 \mathrm{MPa}$. For this particular seismic assessment approach, the maximum bond strength was assumed to be $2.5 \sqrt{f_{c}^{\prime}}$. A cumulative damage indicator of bar strain was estimated using a relationship with the displacement history of the top of the modelled component from response history analysis.

The crack width due to plastic elongation of the reinforcement to be on the order of $10 \mathrm{~mm}$ when the inelastic strain range reached 5 percent 49 . The length of bar yielding is predicted to be the order of $200 \mathrm{~mm}$, which corresponds to $L_{y p}$ being about $3.0 d_{b}$. Overall, the outcomes of the modelling in $49 \mathrm{did}$ indicate that there was no fracture of the reinforcement as the resultant cumulative damage parameter summed to 60 percent of the fatigue life (as predicted based on that particular steel fatigue model and based on a response history analysis using only a single ground motion record).

In the model applied by Davie and Blaikie 49, the extent of crack widening due to plastic elongation of the reinforcement is heavily dependent on the ratio of $f_{u} / f_{y}$ (sometimes denoted $R_{m} / R_{e}$, as in NZS 4671:2001 50). Recent tension tests on deformed bars have shown that relatively high values of $f_{u} / f_{y}$ (of 1.43) are appropriate for Grade 300E reinforcing steel 51 . However, recent tests by the authors have found less extensive strain hardening occurs for Grade 500E reinforcing bars as $f_{u} / f_{y}$ was found to be 1.24 . Furthermore, recent bond pull-out tests 52 have shown the local bond stress may be on the order of $3.0 \sqrt{f_{c}^{\prime}}$ (mean value from 29 pull-out tests of deformed reinforcing bars) and the upper characteristic $95^{\text {th }}$ percentile value was found to be $3.4 \sqrt{f_{c}^{\prime}}$. By considering these probable strength values, as commonly done in practice and according to the NZSEE (2006) guidelines 10, and using the same model prediction equations as 49 , the maximum crack width is reduced by 35 percent and $L_{y p}$ is not greater than $2.0 d_{b}$.

As shown earlier in this paper, Equation 3 from 13 ignores the ratio of $f_{u} / f_{y}$, and instead suggests that $L_{y p}$ is approximately equal to $6.6 d_{b}$ for Grade 300 reinforcement, and $11 d_{b}$ for Grade 500 reinforcement (using $5^{\text {th }}$ percentile values of $f_{y}$ ). Overall, the resulting value of $L_{y p}$ is a significant overestimate compared to the calculations in 49 which used an alternative methodology with a more robust physical meaning. In the calculations shown here by the authors of this paper, Equation 3 vastly over-estimates $L_{y p}$ when Grade $500 \mathrm{E}$ reinforcing steel is used (as in the Gallery Apartments building). In this type of scenario of forming single-crack PHZs, accurate estimation of $L_{y p}$ becomes vitally important for predicting seismic performance at the ultimate limit state.

\section{Dynamic Response of Interacting Structural Systems}

Although the response of individual components has long been studied in experimental testing in laboratories, the interaction between components and their influence on global system response may need further investigation. For instance, there are effects of interactions between floor slabs and beam elongation (NZS3101:2006 6) as axial restraint from floor slabs will increases the strength of adjacent components such as coupling beams in coupled wall systems and beams in moment resisting frames $3,4,53-55$. The axial restraint provided by floor slabs may restrict the formation of diagonal cracks in the web of regular walls. These are some of the reasons that the spread of inelastic deformations in a system of interacting components is likely to deviate from the performance of an individual component. Relative differences in stiffness and the early onset of unexpected yielding can drastically influence the dynamic response and overall performance of a structural system. An example of this behaviour was the Clarendon Tower formerly at 78 Worcester Street, Christchurch 4.

The authors understand there are proposed investigations on the interaction of RC structural systems being undertaking in New Zealand 37. Full scale shake-table or real-time dynamic testing might offer further insight into the behaviour of interacting components; however this type of experimental work is somewhat constrained by the laboratory facilities available in New Zealand. 


\section{Assessing the Residual Capacity of RC Structures}

The issues described in this paper also have implications for practitioners using seismic assessment methods (for damaged and un-damaged evaluations) which are largely based on the assumptions adopted in conventional design practice. Structural engineers have recently been challenged on the subject of evaluating the residual capacity of damaged RC buildings; an issue that also applies to well-designed structures that formed "spreading-PHZs" and performed with sufficient ductility. Uncertainties in the remaining life of the structure are partly due to: (i) the severity and number of strong ground motions in the Canterbury earthquake sequence and subsequent damage to the structure; and (ii) the uncertainty for the effectiveness and cost of structural repair techniques. Hundreds of RC buildings in Christchurch have subsequently been demolished after the Canterbury earthquake sequence.

In both research and in practice, the seismic performance and extent of structural damage is commonly expressed in terms of the peak values of non-cumulative damage indices or "demand metrics" (e.g. peak lateral drift) due to the simplistic nature of obtaining values for these metrics with physical meaning [56] Cumulative demand metrics (e.g. energy-based measures) might provide more reliable information for making postearthquake decisions like seismic retrofit or demolition. Although cumulative metrics are more complicated to determine, they are more important for realistic seismic loading histories where the sequence of loading does not increase monotonically. The use of cumulative demand metrics does however require some calibration of damage model co-efficients (scale factors and exponents). In research these model co-efficients can be determined by using regression techniques based on experimental measurements. In practice however, model co-efficients are not well known as they cannot be determined with the same ease or reliability. Cumulative demand parameters are less comparable between different structural components with in-situ differences such as experimental boundary conditions, specimen geometry, reinforcing content, material properties and different loading protocols. There are current research projects dedicated to the subject of residual capacity of RC structures (38, among others).

\section{CONCLUSION}

This paper was motivated by the unexpected damage to some 'real' RC structures in the 2010-2011 Canterbury earthquakes. The damage to the critical wall in the Gallery Apartments building was described here as a particular example of structural behaviour that is concerning with respect to the "Life-Safety" performance objective at the Ultimate Limit State. One of the main lessons learnt from the Canterbury earthquakes was the concentration of inelastic deformations and brittle failure in some lightly reinforced wall structures which did not compare well to the fanned crack patterns and typical spread of plasticity observed in previous laboratorybased experimental testing.

This paper focussed on differences between common experimental procedures and laboratory conditions and how those vary from the field conditions when a 'real' RC structure is subjected to severe earthquake-induced ground motions. Specific conditions discussed here included the applied loading history and loading rate, the concrete strength and the quantity of longitudinal reinforcement. Increased considerations and changes to structural engineering practice have been promoted by the lessons that have emerged from the Canterbury earthquake sequence. The issues and constraints that were imposed on previous experimental testing may be considered in future research topics to improve the confidence levels for the seismic performance of RC structures at the ultimate limit state.
The latter sections of this paper described an example where practicing structural engineers were challenged to estimate the ductility of a lightly reinforced structural component. The overall results from this example highlighted a major concern that two approaches in the existing literature would give vastly different results for the anticipated level of yield penetration in a single-crack-PHZ.

\section{ACKNOWLEDGMENTS}

The subject of this paper was motivated by many discussions with practising structural engineers throughout New Zealand. Professor Richard Fenwick is acknowledged for discussions on several aspects of this paper and for sharing his experience in previous experimental testing. Comments by Mr. Richard Malcolm on a draft of this paper are acknowledged.

\section{REFERENCES}

1 Kam WY, Pampanin S and Elwood K. (2011). "Seismic Performance of Reinforced Concrete Buildings in the 22 February Christchurch (Lyttelton) Earthquake". Bulletin of the New Zealand Society for Earthquake Engineering; 44(4): 239-278. http://www.nzsee.org.nz/db-/SpecialIssue/44(4)0239.pdf

2 Bull DK (2012). "The Performance of Concrete Structures in the Canterbury Earthquakes: Lessons for Concrete Buildings". Structural Engineers Association of California (SEAOC) Convention, Santa Fe, NM.

3 Canterbury Earthquakes Royal Commission, CERC (2012). "Final report: Volume 2 - The performance of Christchurch CBD Buildings". http://canterbury.royalcommission.govt.nz/Final-Report---Volumes-1-2-and-3

4 Fenwick RC (2013). "Aspects of the Seismic Performance of Concrete Buildings". Conference proceedings of the New Zealand Concrete Society, Queenstown, New Zealand.

5 Fenwick RC and Dhakal RP (2007a). "Material Strain Limits for Seismic Design of Concrete Structures". SESOC Journal, 20(1): 14-28

6 Standards New Zealand. (2006). "NZS3101: Parts 1 and 2 Concrete Structures Standard-New Zealand". Standards New Zealand, Wellington.

7 Walker AF (2007). "Assessment of Material Strain Limits for Defining Different Forms of Plastic Hinge Region in Concrete Structures". Master of Engineering Thesis, University of Canterbury, Christchurch, New Zealand.

8 Beyer K, Dazio A and Priestley MJN (2008). "QuasiStatic Cyclic Tests of Two U-shaped Reinforced Concrete Walls". Journal of Earthquake Engineering, 12:7, 10231053. http://dx.doi.org/10.1080/13632460802003272

9 Priestley MJN and Park R (1984). "Strength and Ductility of Bridge Substructures". RRU Bulletin 71, National Roads Board, Wellington, New Zealand.

10 NZSEE (2006). "Assessment and Improvement of the Structural Performance of Buildings in Earthquakes". Recommendations of a Study Group of the New Zealand Society for Earthquake Engineering. http://www.nzsee.org.nz/db/PUBS/2006_AISPBE_Corrigenda_3\%20.pdf

11 Fenwick RC and Dhakal RP (2007b). "Material Strains and Relevance to Seismic Design". SESOC Journal, 20(1): 5-12.

12 Paulay T and Priestley MJN (1992). "Seismic Design of Reinforced Concrete and Masonry Buildings". John Wiley \& Son, Inc., New York, United States of America.

13 Priestley MJN, Kowalsky MJ and Calvi GM (2007). "Displacement-Based Seismic Design of Structures". IUSS PRESS, Pavia, Italy. 
14 Liddell D, Ingham JM and Davidson BJ (2000). "Influence of Loading History on Ultimate Displacement of Concrete Structures". Report No. 597, University of Auckland, Auckland, New Zealand.

15 Eligehausen R, Popov EP and Bertero VV (1983). "Local Bond Stress-slip Relationships of Deformed Bars under Generalized Excitations". Report UCB/EERC-83/23, University of California, Berkeley. http://nisee.berkeley.edu/documents/EERC/EERC-83-23.pdf

16 Shima H, Chou LL and Okamura H (1987). "Micro and Macro Models for Bond in Reinforced Concrete". Reprinted from Journal of the Faculty of Engineering, The University of Tokyo, 39(2).

17 Bonacci JF (1994a). "Bar Yield Penetration in Monotonically Loaded Anchorages". Journal of Structural Engineering, ASCE, 120(3), 965-986.

18 Mayer U and Eligehausen R (1998). "Bond Behaviour of Ribbed Bars at Inelastic Steel Strains." 2nd International Ph.D. Symposium in Civil Engineering, Budapest.

19 Lowes LN, Moehle JP and Govindjee S (2004). "Concrete-Steel Bond Model for use in Finite Element Modelling of Reinforced Concrete Structures". ACI Structural Journal, 101(4), 501-511. http://dx.doi.org/10.-14359/13336

20 Fédération Internationale du Béton, fib (2012). "Model Code 2010 - Final Draft, Volume 2". FIB Bulletin No. 66, Lausanne, Switzerland.

21 Soleymani Ashtiani M, Dhakal RP and Scott AN (2013) "Post-Yield Bond Behaviour of Deformed Bars in HighStrength Self-compacting Concrete". Construction and Building Materials, 44, 236-248. http://dx.doi.org/10.1016/j.con-buildmat.2013.02.072

22 Dickson AR (1986). "The Response of Reinforced Concrete Slabs to Concentrated Loading”. Report No. 408, University of Auckland, New Zealand.

23 Comite' Euro-internationale du beton, CEB-FIP (1993). "The Model Code 1990: Design code". London: T. Telford; 1993.

24 Standards New Zealand. (1995). "NZS3101: Concrete Structures Standard-New Zealand". Standards New Zealand, Wellington.

25 Smith PC and Devine J (2012a). "Independent Assessment of Earthquake Performance of Craig's Investment Partners House - 90 Armagh Street". Prepared for the Canterbury Earthquakes Royal Commission. http://canterbury.royal-commission.govt.nz/documents-bykey/20120229.3509

26 Smith PC and Devine J (2012b). "Independent Assessment of Earthquake Performance of Inland Revenue Department Building - 224 Cashell Street”. Prepared for the Canterbury Earthquakes Royal Commission. http://canterbury.royal-commission.govt.nz/documents-bykey/20120227.3340

27 Leon RT and Deierlein GG (1996). "Considerations for the use of Quasi-Static Testing". Earthquake Spectra, 12, 87-109.

28 Krawinkler H (2009). "Loading Histories for Cyclic Tests in Support of Performance Assessment of Structural Components". The 3rd International Conference on Advances in Experimental Structural Engineering, October 15-16, San Francisco, CA.

29 Akguzel U and Pampanin S (2010). "Effects of Variation of Axial Load and Bidirectional Loading on Seismic Performance of GFRP Retrofitted Reinforced Concrete Exterior Beam-Column Joints". Journal of Composites for Construction 14(1): 94-104. http://dx.doi.org/10.1061/(ASCE)10900268(2010)14:1(94)
30 Park R (1989). "Evaluation of Ductility of Structures and Structural Assemblages from Laboratory Testing." Bulletin of the New Zealand Society for Earthquake Engineering. 22(3), http://www.nzsee.org.nz/db/Bulletin/Archive/22(3)0155.pdf

31 Bradley BA (2010). "Epistemic Uncertainties in Component Fragility Functions". Earthquake Spectra, 26(1), 41-62. http://dx.doi.org/10.1193/1.3281681

32 FEMA-461 (2007). "Interim Protocols for Determining Seismic Performance Characteristics of Structural and Non-structural Components Through Laboratory Testing”. Prepared by Applied Technology Council (ATC) for Federal Emergency Management Agency (FEMA), Redwood City, California.

33 Vos E and Reinhardt HW (1982). "Influence of Loading Rate on Bond Behaviour of Reinforcing Steel and Prestressing Strands". Materials and Structures. RILEM 15(85), 3-10.

34 Chung L and Shah SP (1989). "Effect of Loading Rate on Anchorage Bond and Beam-Column Joints." ACI Structural Journal, 86(2), 132-142. http://dx.doi.org/10.14359/2628

35 Phan V, Saiidi MS, Anderson J and Ghasemi H (2007). "Near-Fault Ground Motion Effect on Reinforced Concrete Bridge Columns". Journal of Structural Engineering, ASCE, 133(7), 982-989. http://dx.doi.org/10.1061/(ASCE)0733-9445(2007)133:7(982)

36 Choi H, Saiidi MS, Somerville P and El-Azazy S (2010). "Experimental Study of Reinforced Concrete Bridge Columns Subjected to Near-Fault Ground Motions". ACI Structural Journal, 107(1), 3-12. http://dx.doi.org/10.14359/51663383

37 Bennett A, Sharpe RD, and Stannard MC (2014). "Prioritising Research into the Seismic Performance of Reinforced Concrete." Conference Proceedings of the New Zealand Society of Earthquake Engineering, Auckland. http://db.nzsee.org.nz/2014/oral/38_Bennett.pdf

38 Cuevas A and Pampanin S (2015). "Effect of Strain-Rate and Material Characteristics on the Seismic Residual Capacity of Reinforced Concrete Plastic Hinges: Numerical Investigation." Conference proceedings of the New Zealand Society of Earthquake Engineering, Rotorua.

39 Holmes Solutions (2011). "Materials Testing in Buildings of Interest: Prepared for the Canterbury Earthquakes Royal Commission." November 2011, Christchurch, New Zealand. http://canterbury.royalcommission.govt.nz/documents-by-key/20111129.1351

40 Gopalaratnam VS and Shah SP (1985). "Softening Response of Plain Concrete in Direct Tension". ACI Journal, 82(3), 310-323. http://dx.doi.org/10.14359/10338

41 Henry RS (2013). "Assessment of Minimum Vertical Reinforcement Limits for RC walls." Bulletin of the New Zealand Society for Earthquake Engineering. 46(2), 8896. http://www.nzsee.org.nz/db/Bulletin/Current/46(2)0088.pdf

$42 \mathrm{Lu} \mathrm{Y,} \mathrm{Henry} \mathrm{RS} \mathrm{and} \mathrm{Ma} \mathrm{QT} \mathrm{(2014).} \mathrm{"Numerical}$ Modelling and Testing of Concrete Walls with Minimum Vertical Reinforcement". Conference proceedings of the New Zealand Society of Earthquake Engineering, Auckland. http://db.nzsee.org.nz/2014/oral/23_Lu.pdf

43 Henry RS, Lu Y and Ingham JM (2014). "Experimental Testing and Modelling to Address the Performance of RC Walls during the 2010/2011 Canterbury Earthquakes". Proceedings of the 10th National Conference in Earthquake Engineering, Earthquake Engineering Research Institute, Anchorage, AK. 
44 Fenwick RC and Sue CFC (1982). "The Influence of Water Gain upon the Tensile Strength of Concrete". Magazine of Concrete Research, 34(120): 139-145.

45 SESOC (2011). "Preliminary Observations from Christchurch Earthquakes". Prepared for the Canterbury Earthquakes Royal Commission. http://canterbury.royalcommission.govt.nz/documents-by-key/20111205.1533

46 Soleymani Ashtiani M, Dhakal RP and Scott, AN (2014). "Seismic Performance of High-Strength Self-Compacting Concrete in Reinforced Concrete Beam-Column Joints." Journal of Structural Engineering, ASCE, 140(5), 04014002. http://dx.doi.org/10.1061/(ASCE)ST.1943$\underline{541 X .0000973}$

47 SESOC (2012). "Practice note - Design of Conventional Structural Systems Following Canterbury Earthquakes". Prepared for the Canterbury Earthquakes Royal Commission. http://canterbury.royalcommission.govt.nz/documents-by-key/20111221.1908

48 Sritharan S, Beyer K, Henry RS, Chai YH, Kowalsky MJ and Bull DK (2014). "Understanding Poor Seismic Performance of Concrete Walls and Design Implications". Earthquake Spectra, 30(1), 307-334. http://dx.doi.org/10.1193/021713EQS036M

49 Davey RA and Blaikie EL (2005). "On the Flexural Ductility of very Lightly Reinforced Concrete Sections". Conference proceedings of the New Zealand Society of Earthquake Engineering, Taupo. http://www.nzsee.org.nz/db/2005/-Paper15.pdf

50 Standards New Zealand (2001). "NZS4671: Steel Reinforcing Materials Standard-New Zealand.” Standards New Zealand, Wellington.

51 Davies-Colley S, Kleinjan B, Bull DK and Morris GJ (2015). "Review of the Material and Flexural Overstrength Factors for Grade 300E Reinforcing Steel used in New Zealand". Conference proceedings of the New Zealand Society of Earthquake Engineering, Rotorua, New Zealand.

52 Morris GJ, Bull DK and Bradley BA, (2015). "Monotonic and Cyclic Bond Behaviour of Deformed Bars in Reinforced Concrete Structures". Conference proceedings of the New Zealand Society of Earthquake Engineering, April 2015, Rotorua, New Zealand.

53 Lau DBH (2007). "Influence of Precast Prestressed Flooring on Seismic Performance of Reinforced Concrete Perimeter Frame Buildings". Doctor of Philosophy Thesis, University of Auckland, New Zealand.

54 Malcolm RC, Bull DK, Henry RS and Ingham JM (2014). "The Effects of Axial Restraint in Reinforced Concrete Coupling Beams." Proceedings of the New Zealand Concrete Industry Conference, Taupo.

55 Naish D, Fry JA, Klemencic R and Wallace J (2009). "Experimental Evaluation and Analytical Modeling of ACI 318-05/08 Reinforced Concrete Coupling Beams Subjected to Reversed Cyclic Loading”. UCLA/SGEL Report-2009/06, University of California, Los Angeles.

56 Williams MS and Sexsmith RG (1995). "Seismic Damage Indices for Concrete Structures: A State-of-the-art Review". Earthquake Spectra, 11(2), 319-349. $\underline{\text { http://dx.doi.org/10.1193/1.1585817 }}$ 